\title{
Ação fisioterapêutica na recuperação do paciente com acidente vascular encefálico: uma revisão bibliográfica
}

As condições econômicas e socioculturais possuem ligação direta com a predisposição para o surgimento de doenças crônicas não transmissíveis, e dentre elas, o acidente vascular encefálico. O presente estudo tem como objetivo analisar os impactos dos exercícios terapêuticos neurofuncionais nos pacientes pós-Acidente Cerebral Encefálico. Trata-se de uma revisão bibliográfica qualitativa, descritiva e exploratória. A análise partiu da leitura de artigos publicados em periódicos no período de 2009 a 2018, observando as principais técnicas de tratamentos fisioterapêuticos. Foram encontrados um total de 41 artigos, sendo que 20 artigos tratavam do AVE, 13 artigos tratavam do papel do fisioterapeuta na restauração de pacientes e apenas 8 expunham sobre o papel do fisioterapeuta na reabilitação de doentes vítimas de AVE. Dos 8 artigos, 5 foram utilizados para a revisão, sendo que 36 foram descartados por se inserir em um ou mais processos de eliminação. As principais técnicas que surtiram efeito na doença estão o treino de marcha na esteira com (SPP), Bandagem Funcional associada ou não a FES (Estimulação Elétrica Funcional) e vibração na dorsiflexão e descarga de peso, Hidroterapia com o método de Bad Ragaz, o TENS (Estimulação Elétrica Nervosa Transcutânea) e Terapia de Contensão Induzida (TCl).

\section{Physical therapy action in the recovery of patients with stroke: a bibliographic review}

\begin{abstract}
Economic and sociocultural conditions are directly linked to a predisposition to the emergence of noncommunicable chronic diseases, and among them, stroke. The present study aims to analyze the effects of neurofunctional therapeutic exercises in post-stroke patients. This is a qualitative, descriptive and exploratory literature review. A partial analysis of the reading of articles published in journals from 2009 to 2018 , observing as the main techniques of physical therapy application. A total of 41 articles were found, of which 20 articles dealt with in the stroke, 13 articles dealt with in the role of physiotherapist in the restoration of patients and only 8 stated about the role in the physiotherapist in the rehabilitation of stroke patients. Of the 8 articles, 5 were used for review, 36 were discarded for inserting one or more replacement processes. The main techniques that affect the effect of the disease or treadmill gait training (SPP), Functional Bandage with or without FES (Functional Electrical Stimulation) and vibration in dorsiflexion and weight discharge, Bad Ragaz Hydrotherapy, or TENS (Transcutaneous Electrical Nerve Stimulation) and Induced Containment Therapy (TCI).
\end{abstract}

Keywords: Noncommunicable Chronic Diseases; Mortality; Leakage; Physiotherapy.

Topic: Fisioterapia

Reviewed anonymously in the process of blind peer
Received: 05/04/2019

Approved: 07/07/2019
Nayane Mendes Lopes

Faculdade Guaraí, Brasil

http://lattes.cnpq.br/9421423227309572

nayanemendes776@gmail.com

Raimunda Noronha Aguiar

Faculdade Guaraí, Brasil

http://lattes.cnpq.br/1355099749029186

meythyaguiarsantos@hotmail.com

Aluísio Vasconcelos de Carvalho (ic)

Faculdade Guaraí, Brasil

http://lattes.cnpq.br/5200758055263996

http://orcid.org/0000-0002-3793-3133

aluisiovasconcelos@gmail.com
Referencing this:

LOPES, N. M.; AGUIAR, R. N.; CARVALHO, A. V.. Ação fisioterapêutica na recuperação do paciente com acidente vascular encefálico: uma revisão bibliográfica. Medicus, v.1, n.2, p.1-8, 2019. DOI: http://doi.org/10.6008/CBPC2674-6484.2019.002.0008 


\section{INTRODUÇÃO}

As mudanças nos paradigmas sociais diante o aumento da expectativa de vida, juntamente com a progressiva inversão da pirâmide etária, têm proporcionado uma alteração no perfil epidemiológico em escala nacional, no qual afeta diretamente as causas de mortalidade, retraindo o aparecimento de doenças infectocontagiosas e proporcionando o aumentando da manifestação de doenças crônicas não transmissíveis (DCNT) (ARAÚJO et al., 2018; SILVA et al., 2014).

As condições econômicas e socioculturais possuem ligação direta com a predisposição para o surgimento de DCNT. Segundo Malta et al. (2013), a parcela da população afetada pelas doenças crônicas não transmissíveis estão localizadas nas áreas mais pobres e menos desenvolvidas, no qual há um baixo nível de escolarização e, consequentemente, há uma maior dificuldade ao acesso à informação e a serviços sociais e de saúde, deixando claro as consequências das desigualdades sociais existentes nos diversos setores da sociedade.

As DCNT são umas das maiores causas de mortes no mundo, sendo que essas afetam mais de $50 \%$ da população mundial acima dos 50 anos de idade (AZEVEDO et al., 2014). Dentre as DCNT, o Acidente Vascular Encefálico (AVE) atinge cerca 16 milhões de pessoas por ano, propiciando uma taxa de 5,7 milhões de mortes, ocorrendo principalmente em pessoas de idade avançada. A Hipertensão Arterial Sistêmica (HAS), doença crônica não transmissível, é considerada uma das DCNT que possuem uma das maiores taxas de ocorrência mundial, com estimativa de $37,8 \%$ entre os homens e $31 \%$ nas mulheres, considerada um problema de alto grau por elevar os riscos de desenvolvimento de acidente vascular encefálico (CARVALHO et al., 2015; GONÇALVES et al., 2019).

Aproximadamente 15\% dos casos de Acidente Vascular Encefálico Hemorrágico (AVEH) e 85\% dos casos de Acidente Vascular Encefálico Isquêmico (AVEI) são decorrentes do não tratamento, ou tratamento de maneira inadequada do HAS (CAVALCANTE et al., 2010; MENDES et al., 2014). No Brasil, a Hipertensão Arterial Sistêmica possui taxas superiores a 30\% em ambos os sexos (SPENCE, 2013; ARAÚJO et al., 2017).

O Acidente Vascular Encefálico é caracterizado pela interrupção da circulação sanguínea no encéfalo, ocorre quando há alguma deficiência na realização das ações fisiológicas normais do sistema nervoso que, por consequência, ocasionam uma oclusão ou obstrução de vasos sanguíneos, ou ainda, formação de coágulos sanguíneos que diminuem a oxigenação do encéfalo e distribuição de nutrientes ocasionando necrose encefálica, podendo levar a diversas complicações fisiológicas e perdas de funções (GARRITANO et al., 2012; MOREIRA et al., 2010).

O AVE é subdividido em dois tipos distintos: Acidente Vascular Encefálico Isquêmico (AVEI) e o Acidente Vascular Encefálico Hemorrágico (AVEH). O Acidente Vascular Encefálico Isquêmico é o tipo mais comum, ocorrendo quando há oclusão da circulação do sanguínea (isquemia) em consequência da formação de embolia sendo originária do coração.

O AVEI pode ocorrer também através da oclusão de pequenas artérias ou ainda a ocorrência de tromboses de grandes vasos sanguíneos. Já o Acidente Vascular Hemorrágico é caracterizado pelo 
rompimento de algum vaso sanguíneo cerebral, no qual há o extravasamento do tecido sanguíneo em alguma localidade do sistema nervoso (FREITAS et al., 2016; LIMA et al., 2016a, 2016b). A Organização Mundial da Saúde classifica o AVE como a presença de sinalização de distúrbios progressivos nas funções cerebrais, podendo levar a morte, sem que haja nenhuma outra razão aparente além da obstrução vascular encefálica.

Nos últimos anos, o conhecimento científico obteve grandes avanços a respeito do entendimento das causas e tratamentos do Acidente Vascular Cerebral. Em relação ao tratamento, as descobertas mostraram a importância dos cuidados iniciais que, se possível, devem ocorrer ainda durante a fase aguda do AVE, a fim de diminuir os danos, ou evitar lesões cerebrais permanentes (MANIVA et al., 2013). Nesse sentido, o diagnóstico, por meio da ressonância magnética, tomografia computadorizada ou exame neurológico para especificação do AVE ocorrente no paciente é de extrema importância, pois é a partir desse ponto que será possível determinar o tipo de tratamento que o paciente deverá receber (CRUZ, 2012).

Os fatores de risco que podem expor uma pessoa ao desenvolvimento de um AVE são multifatoriais, variando de indivíduo para indivíduo; a hipertensão arterial, por exemplo, é um dos principais fatores de riscos que, por estar associados a enfermidade de grandes e pequenas artérias, elevam os índices para o desenvolvimento de AVE (ASSIS et al., 2018; LOPES et al., 2016). Além de Hipertensão arterial, há diversos outros fatores de riscos envolvido com o aparecimento de AVE, tais como doenças cardíacas, tabagismo, doença vascular prévia, diabetes, uso excessivo de drogas, sedentarismo, obesidade; anticoncepcionais (GOLDSTEIN et al., 2011). Além dessas, há fatores que independem do estilo de vida do indivíduo, tais como sexo, idade, raça e fatores hereditários (RODRIGUES et al., 2013; AZEVEDO et al., 2018).

De acordo com World Stroke Organization, os sinais de um Acidente Vascular Encefálico podem ser reconhecidos facilmente por qualquer indivíduo. Dentre os principais sintomas estão a paralisia facial, dificuldades na fala, enfraquecimento dos músculos dos braços, confusão mental, distúrbio de visão, alteração de equilíbrio e coordenação motora, tonturas, dores de cabeça de maneira intensa e súbita (COSTA, 2009).

É de extrema importância que, ao detectar a ocorrência de AVE, o paciente seja hospitalizado de maneira emergencial sendo severamente monitorado nas primeiras horas após a ocorrência e posteriormente, a fim de analisar a magnitude dos danos causados (HACKE et al., 2017). No Brasil, o Acidente Vascular Encefálico é uma das principais razões de internações, deficiência e mortalidade, atingido aproximadamente cerca de $90 \%$ de indivíduos com alguma disfunção total ou parcial (hemiplegia ou hemiparesia) (FERREIRA et al., 2018). Diante do exposto, surgiram os seguintes questionamentos que nortearam a realização do presente trabalho: 'Qual a incidência de Acidente Vascular Encefálico no Brasil?' e 'Qual o papel do fisioterapeuta na reabilitação do paciente que desenvolveu um AVE?'.

Em razão da necessidade de se discutir os avanços científicos em relação ao Acidente Vascular Encefálico, bem como contribuir com o desenvolvimento científico e medicinal, o presente trabalho justificase em razão de procurar compreender as maiores dificuldades enfrentadas no Brasil em relação ao alto índice da doença e seus agravos.

Nesse sentido, o presente artigo objetivou-se em discutir, por meio de revisão bibliográfica, a 
incidência de Acidente Vascular Encefálico no Brasil e a ação fisioterapêutica na reabilitação do paciente, bem como os fatores que geram risco para a saúde no qual aumentam o índice de morbidade e mortalidade, além de enfatizar a importância de hábitos saudáveis para a manutenção da saúde física e mental.

\section{METODOLOGIA}

O presente trabalho trata-se de uma revisão bibliográfica, no qual avaliou de maneira qualitativa, descritiva e exploratória, o Acidente Vascular Encefálico no Brasil. Assim, para o desenvolvimento da seguinte pesquisa, foi realizado um levantamento de artigos indexados nas bases de dados Scielo, LILACS, CAPES, Bireme, Google Acadêmico, Biblioteca Virtual em Saúde (BVS) e livros.

De acordo com as normativas de Marcone et al. (2010), determina-se que a pesquisa de revisão bibliográfica deve ser realizada em três etapas, de maneira sistemática e objetiva. Assim, segundos Marcone et al. (2010), a primeira etapa da pesquisa de revisão bibliográfica consiste na delimitação do tema a ser pesquisado, onde ele deve apresentar relevância científica e social, buscando a resolução de um determinado problema ou questionamento.

A segunda etapa configura-se na busca por materiais atualizados que vão constituir as fontes de informação a serem discutidas. A terceira e última etapa, consiste na elaboração do documento, onde todas as informações relevantes encontradas na segunda etapa necessitam estar determinada de maneira lógica e conforme a relação do tema selecionado. Utilizou-se os seguintes critérios de inclusão: artigos publicados recentemente; população alvo de pacientes pós-AVE, levando em consideração as fases subagudas e crônicas determinadas pelos respectivos artigos, sendo que as intervenções utilizadas foram a fisioterapêutica convencional; as intervenções mais eficientes; e faixa etária associada ao grupo de risco e no amostral.

\section{RESULTADOS E DISCUSSÃO}

Foram encontrados um total de 41 artigos, sendo que 20 artigos tratavam do AVE, 13 artigos falavam do papel do fisioterapeuta na reabilitação de doentes, e apenas 8 descreviam sobre o papel do fisioterapeuta na recuperação de pacientes vítimas de AVE. Dos 8 artigos, 5 foram utilizados para a revisão sistêmica, sendo que 36 foram descartados por se encaixar em um ou mais critérios de exclusão. As características de cada estudo são verificadas no Quadro 1.

A partir desses resultados foram constatados que a fisioterapia teve eficácia nos artigos analisados por Garção et al. (2017), Guimarães et al. (2015), Miranda et al. (2018), Pompeu et al. (2014) e Siqueira et al. (2013), obtendo resultados através de protocolos de tratamento fisioterapêutico convencional, avaliando e observando que os fatores limitantes do paciente pós-AVE foi essencial para uma melhor abordagem terapêutica, visto que qualquer intervenção deve ser direcionada a todos os aspectos que envolvem as disfunções.

No primeiro momento, após a ocorrência do Acidente Vascular Cerebral, o indivíduo pode apresentar modificações no tônus muscular em que o lado oposto do hemisfério cerebral afetado, o dimídio possivelmente estará em estado caracterizado por flacidez, que impede a estabilização do ombro do paciente 
em qualquer posição (MARCOTTI, 2005). Assim, poderá acarretar ao paciente a supressão de movimentos do lado corporal afetado, no qual será responsável por gerar diversos mecanismos compensatórios como algumas complicações secundárias: subluxação do ombro, encurtamentos miogênicos, síndrome ombromão, síndrome de Pusher, deformidades e edema de mão (FREITAS, 2006).

Quadro 1: Características dos estudos avaliados.

\begin{tabular}{|c|c|c|c|c|}
\hline Autores & Intervenção & $\begin{array}{c}\text { № } \\
\text { amostral }\end{array}$ & $\begin{array}{l}\text { Faixa } \\
\text { etária }\end{array}$ & Resultados \\
\hline $\begin{array}{l}\text { Garção et al. } \\
\text { (2017) }\end{array}$ & $\begin{array}{l}\text { O treino de marcha na } \\
\text { esteira com (SPP). }\end{array}$ & 42 & $\begin{array}{l}45 a \\
75\end{array}$ & $\begin{array}{l}\text { Preveniu o fenômeno do não uso e a prática da marcha sem medo e } \\
\text { quedas. }\end{array}$ \\
\hline $\begin{array}{l}\text { Guimarães et } \\
\text { al. (2015) }\end{array}$ & $\begin{array}{l}\text { Bandagem Funcional } \\
\text { associada ou não } \\
\text { a FES (Estimulação Elétrica } \\
\text { Funcional) e vibração na } \\
\text { dorsiflexão e descarga de } \\
\text { peso. }\end{array}$ & 7 & $\begin{array}{l}40 a \\
70\end{array}$ & Reestruturou a plasticidade neural. \\
\hline $\begin{array}{l}\text { Miranda et } \\
\text { al. (2018) }\end{array}$ & $\begin{array}{l}\text { Hidroterapia com o método } \\
\text { de Bad Ragaz. }\end{array}$ & 13 & $\begin{array}{l}48 a \\
75\end{array}$ & $\begin{array}{l}\text { Ocorreu a Retração do tônus muscular, elevação da angulação articular, } \\
\text { relaxamento, fortalecimento muscular, restabelecimentos e } \\
\text { normatização dos padrões de movimentos dos membros inferiores e } \\
\text { superiores, fortificação muscular e preparação dos membros inferiores. }\end{array}$ \\
\hline $\begin{array}{l}\text { Pompeu et } \\
\text { al. (2014) }\end{array}$ & $\begin{array}{l}\text { TENS (Estimulação Elétrica } \\
\text { Nervosa Transcutânea) }\end{array}$ & 13 & $\begin{array}{l}41 a \\
80\end{array}$ & Melhora da anomalia do tônus e da ação do reflexo fásico de constrição. \\
\hline $\begin{array}{l}\text { Siqueira et } \\
\text { al. (2013) }\end{array}$ & $\begin{array}{l}\text { Terapia de Contensão } \\
\text { Induzida (TCl) }\end{array}$ & 20 & $\begin{array}{l}45 a \\
75\end{array}$ & $\begin{array}{l}\text { Ganho de força muscular, aumento da amplitude dos movimentos, } \\
\text { aumento de sensibilidade nos membros superiores e aumento da } \\
\text { mobilidade dos membros afetados. }\end{array}$ \\
\hline
\end{tabular}

De acordo com Costa (2017) a consequência mais ocorrente de um AVC é a hemiparesia estática, caracterizada pela elevação do aumento da resistência passiva ao alongamento, através do aumento do tônus muscular, em que envolve hiperreflexia e hipertonia elástica no momento da contração muscular, o que provoca uma situação neurológica anormal e sinaliza existência de Babinski positivo e clônus. A espasticidade do indivíduo que possui hemiparesia implica majoritariamente a ação dos músculos antigravitacionais, o que por consequência, causa alteração na postura flexora do membro superior e extensora de membro inferior agredido (WISSEL et al., 2013).

Desse modo, a ação fisioterapêutica é de extrema importância para reabilitação do paciente acometido por um AVC, seja na fase aguda ou na fase crônica, sendo de responsabilidade do profissional avaliar o quadro do paciente e apresentando um programa terapêutico de acordo com suas necessidades e limitações (BRANCO et al., 2010).

O tratamento fisioterapêutico, procura coibir a ação reflexa patológica em função de adequar o tônus muscular e retrair a ação dos movimentos normais. Entretanto, cada tratamento deve ser direcionado a fisiopatologia específica do paciente, ou seja, o tratamento deve variar dependendo da área e intensidade afetada (SOUZA et al., 2012; BRASIL, 2012).

Dentre os tratamentos existentes, a hidroterapia é uma das maneiras de promover a recuperação do paciente, em que procura proporcionar a independência funcional do mesmo através dos privilégios físicos e termodinâmicos da água (MENEGHETTI et al., 2012). Dentre as técnicas que podem ser utilizadas na hidroterapia estão o método de Bad Ragaz, que objetiva o retração do tônus muscular, elevação da angulação articular, relaxamento, fortalecimento muscular, restabelecimentos e normatização dos padrões de movimentos dos membros inferiores e superiores, fortificação muscular e preparação dos membros 
inferiores para o carregamento do peso corporal (MIRANDA et al., 2018).

A utilização do TENS em pacientes acometidos por AVC no tratamento da espasticidade pode contribuir na reabilitação de funcional dos movimentos através da supressão da anomalia do tônus e da ação do reflexo fásico de constrição (POMPEU et al., 2014); A Estimulação Elétrica Funcional (SEG), Terapia Vibratória (TV) e Bandagem Funcional (BF) podem ser utilizados para a reabilitação funcional de estruturas do dimídio por meio da reestruturação da plasticidade neural (GUIMARÃES et al., 2015).

Outro método altamente utilizado é o treino de marcha na esteira com SPP, em que objetiva o indivíduo a praticar parâmetros tempo inter e intramembros, além de permitir a condução dos membros afetados antes que o paciente tenha força muscular necessária para conseguir suportar o seu peso corporal. Outra vantagem do treino de marcha na esteira com SSP é que o paciente vai ganhando confiança e evitando a ocorrência de fenômenos como o "não uso aprendido dos membros", quando aplicado de maneira precoce, auxilia na evitabilidade da atrofiação muscular, a perda de força e ajuda no equilíbrio corporal pelo reforço e sustentação de pesos (GARÇÃo et al., 2017).

A Terapia por Contensão Induzida (TCl) ou Técnica de Restrição, possui como objetivo a recuperação dos membros superiores hemiparéticos em pacientes que sofreram alguma lesão cerebral e que tiveram sequelas motoras adquiridas (SIQUEIRA et al., 2013). Apesar de ser inicialmente direcionada a reabilitação dos membros superiores, a TCl já foi descrita, em algumas literaturas, como uma terapia que obteve eficácia na recuperação de membros inferiores. Desse modo, a $\mathrm{TCl}$ é direcionada a membros específicos que estão em desuso e que sofreram lesão estando estes na fase crônica. As ações da TCl são voltadas para o ganho de força muscular, aumento da amplitude dos movimentos, aumento de sensibilidade nos membros superiores e aumento da mobilidade dos membros afetados (SIQUEIRA et al., 2013).

\section{CONSIDERAÇÕES FINAIS}

Diante do exposto acima, fica perceptível a importância clínica e social do acidente vascular encefálico a medida em que acometem milhares de vidas por ano e as projeções de ocorrência dessa doença para o futuro são altamente alarmantes, assim como também fica nítido a necessidade de uma reeducação social referente ao estilo de vida em virtude de procurar diminuir os índices de ocorrência de AVC.

Outro ponto muito importante é a necessidade de melhorar o acesso de pacientes com lesões adquiridas ao tratamento fisioterapêutico, as pesquisas científicas mostram a eficácia dos tratamentos fisioterapêuticos na recuperação e reabilitação de pacientes que possuem alguma sequela acometida por um acidente vascular encefálico. Além disso, deve-se ressaltar que, em detrimento da quantidade de técnicas existentes, cabe ao profissional fisioterapeuta analisar a situação em que o indivíduo se encontra e procurar os melhores programas de tratamentos em que melhor se adequa as necessidade e limites do paciente.

\section{REFERÊNCIAS}

ARAÚJO, J. P. D.; DARCIS, J. V. V.; TOMAS, A. C. V.; MELLO, W. A. D.. Tendência da mortalidade por acidente vascular cerebral no município de Maringá, Paraná entre os anos de
2005 a 2015. International Journal of Cardiovascular Sciences, v.31, n.1, p.56-62, 2018. 
ARAUJO, L. P. G.; SOUZA, G. S.; DIAS, P. L. R.; NEPOMUCENO, R. M.; COLA, C. S. D.. Principais fatores de risco para acidente vascular encefálico e suas consequências: uma revisão de literatura. Pensamento Científico, v.3, n.1, p.283-296, 2017.

ASSIS, C. C. A.; SILVA, J. A. S.; PAIVA, J. T. S.; COUTO, L. B.; SOUZA, S. S. F.; CAMPOS, L. P. F.. Agravos e riscos à saúde do idoso relacionado ao sistema cardiovascular: hipertensão arterial e acidente vascular cerebral. Biológicas \& Saúde, v.8, n.27, 2018.

AZEVEDO, E. C. D. C.; DIAS, F. M. R. D. S.; DINIZ, A. D. S.; CABRAL, P. C.. Consumo alimentar de risco e proteção para as doenças crônicas não transmissíveis e sua associação com a gordura corporal: um estudo com funcionários da área de saúde de uma universidade pública de Recife (PE), Brasil. Ciência \& Saúde Coletiva, v.19, p.1613-1622, 2014.

AZEVEDO, G. V. O.; ARAÚJO, A. H. V.; SOUZA, T. A.. Aspectos epidemiológicos do acidente vascular encefálico na Paraíba em 2016. Fisioterapia Brasil, v.19, n.5, p.236-241, 2018.

BRANCO, T.; SANTOS, R.. Reabilitação da Pessoa com AVC. Coimbra: Formasau, 2010.

BRASIL. Ministério da Saúde. Linha de Cuidados em Acidente Vascular Cerebral (AVC) na rede de Atenção às Urgências e Emergências. Brasília: MS, 2012.

CARVALHO, M. I. F.; DELFINO, J. A. S.; PEREIRA, W. M. G., MATIAS, A. C. X.; SANTOS, E. F. S.. Acidente Vascular Cerebral: Dados clínicos e epidemiológicos de uma clínica de fisioterapia do sertão nordestino Brasileiro. Interfaces: Saúde, Humanas e Tecnologia, v.2, n.6, 2015.

CAVALCANTE, T. F.; MOREIRA, R. P.; ARAÚJO, T. L.; OLIVEIRA LOPES, M. V.. Fatores demográficos e indicadores de risco de acidente vascular encefálico: comparação entre moradores do município de Fortaleza e o perfil nacional. Revista LatinoAmericana de Enfermagem, v.18, n.4, 2010.

COSTA, H. J. B.. Análise cinemática de pacientes com acidente vascular cerebral durante jogo de dardos em ambientes virtual e real. Dissertação (Mestrado em Fisioterapia) - Universidade Federal do Rio Grande do Norte, Natal, 2017.

COSTA, J. H. C.. AVC e diabetes Mellitus: o perfil dos doentes e do AVC. Dissertação (Mestrado Integrado em Medicina) - Universidade da Beira Interior, Covilhã, 2009.

CRUZ, D. M. C.. Terapia ocupacional na reabilitação pósacidente vascular encefálico. Santos: 2012.

FERREIRA, K.; MALCHER, A.; NASCIMENTO, A. P.. Efeitos da terapia por realidade virtual em pessoas que sofreram um acidente vascular encefálico - revisão de literatura. Arquivos Catarinenses de Medicina, v.47, n.3, p.197-203, 2018.

FREITAS, A. S.; COSTA, F. J. V.; SOUSA, I. B.; SOUZA, R. M.; CARNEIRO, R. V.; MANIVA, S. J. C. F.. Jogo educativo sobre acidente vascular cerebral para pré-Adolescentes. Mostra Interdisciplinar do Curso de Enfermagem, v.2, n.2, 2016.

FREITAS, M. S.. A atenção básica como campo de atuação da fisioterapia no Brasil: as diretrizes curriculares resignificando a prática profissional. Rio de Janeiro: UERJ,
2006.

GARÇÃO, D. C.; SOBRAL, J. M.; SANTOS, J. C. D.; MENDONÇA, V. B. D.; REZENDE, L. S.; SANTANA, M. M. S.; FONTES, P. A.; OLIVEIRA, T. V. C.. Intervenção fisioterapêutica com suporte parcial de peso em indivíduos pós-ave: revisão sistemática. Scire Salutis, v.7, n.1, p.6-14, 2017. DOI: http://doi.org/10.6008/SPC2236-9600.2017.001.0001

GARRITANO, C. R.; LUZ, P. M.; PIRES, M. L. E.; BARBOSA, M. T. S.; BATISTA, K. M.. Análise da tendência da mortalidade por acidente vascular cerebral no Brasil no século XXI. Arquivos Brasileiros de Cardiologia, v.98, n.6, 2012.

GOLDSTEIN, L. B.; BUSHNELL, C. D.; ADAMS, R. J.; APPEL, L. J.; BRAUN, L. T.; CHATURVEDI, S.; HINCHEY, J. A.. Guidelines for the primary prevention of stroke: a guideline for healthcare professionals from the American Heart Association / American Stroke Association. Stroke, v.42, n.2, p.517-584, 2011.

GONÇALVES, J. L.; FEITOSA, E. S.; BORGES, R. T.. Perfil epidemiológico de vítimas de acidente vascular encefálico em um hospital de referência do Ceará / Brasil. Interdisciplinar, v.12, n.2, p.92-103, 2019.

GUIMARÃES, S. S.; FERREIRA, D. M.; SILVA, A. M.; SILVA, A. T.; KOSOUR, C.; REIS, L. M.. Efeito da Bandagem Funcional associada ou não à FES e vibração na dorsiflexão e descarga de peso pós-AVC. Neurociências, v.23, n.3, p.383-389, 2015

HACKE, W.; DAVIS, S.. Organizational Update: World Stroke Organization. Stroke, v.48, n.7, p.157-158, 2017.

LIMA, A. C. M. A. C. C.; SILVA, A. L.; GUERRA, D. R.; BARBOSA, I. V.; BEZERRA, K. C.; ORIÁ, M. O. B.. Diagnósticos de enfermagem em pacientes com acidente vascular cerebral: revisão integrativa. Revista Brasileira de Enfermagem, v.69, n.4, p.785-792, 2016a.

LIMA, M. J. M. R.; MOREIRA, T. M. M.; FLORÊNCIO, R. S.; NETO, P. B.. Fatores associados ao conhecimento dos adultos jovens sobre histórico familiar de Acidente Vascular Cerebral. Revista Latino-Americana de Enfermagem, v.24, p.1-9, 2016b.

LOPES, J. M.; SANCHIS, G. J. B.; MEDEIROS, J. L. A. D.; DANTAS, F. G.. Hospitalização por acidente vascular encefálico isquêmico no Brasil: estudo ecológico sobre possível impacto do Hiperdia. Revista Brasileira de Epidemiologia, v.19, p.122-134, 2016.

MALTA, D. C.; SILVA, J. B.. O Plano de Ações Estratégicas para o Enfrentamento das Doenças Crônicas Não Transmissíveis no Brasil e a definição das metas globais para o enfrentamento dessas doenças até 2025: uma revisão. Epidemiologia e Serviços de Saúde, v.22, n.1, p.151-164, 2013.

MANIVA, S. J. C. F.; FREITAS, C. H. A.; JORGE, M. S. B; CARVALHO, Z. M. F.; MOREIRA, T. M. M.. Vivendo o acidente vascular encefálico agudo: significados da doença para pessoas hospitalizadas. Revista da Escola de Enfermagem da USP, v.47, n.2, p.362-368, 2013.

MARCONI, M. A.; LAKATOS, E. M.. Metodologia científica. 3 ed. São Paulo: Atlas, 2010. 
MARCOTTI, A. C.. Estudo dos efeitos da dominância hemisférica cerebral esquerda sobre o acidente vascular encefálico através de três escalas de funcionalidade. Cascavel: Universidade Estadual do Oeste do Paraná, 2005.

MENDES, G. S.; MORAES, C. F.; GOMES, L.. Prevalência de hipertensão arterial sistêmica em idosos no Brasil entre 2006 e 2010. Revista Brasileira de Medicina de Família e Comunidade, v.9, n.32, p.273-278, 2014.

MENEGHETTI, C. H. Z.; CARRARO, L.; LEONELLO, L. A.; BATISTELLA, A. C. T.; JÚNIOR, L. C. F.. A Influência da Fisioterapia Aquática na Função e Equilíbrio no Acidente Vascular Cerebral. Neurociencias, v.20, n.3, p.410-414, 2012.

MIRANDA, M. R.; BUENO, G. C. R.; RIBEIRO, L. C.; MATOS, J. F. S.; FONSECA, C. F.. Benefícios da hidroterapia em pacientes após acidente vascular cerebral (AVC). Revista de Iniciação Científica e Extensão, v.1, n.5, p.465-471, 2018.

MOREIRA, R. P.; ARAÚJO, T. L.; CAVALCANTE, T. F.; GUEDES, N. G.; LOPES, M. V.; COSTA, A. G. S; CHAVES, D. B. R.. Acidente vascular encefálico: perfil de indicadores de risco. Revista da Rede de Enfermagem do Nordeste, v.11, n.2, p.121-128, 2010

POMPEU, J. E.; POMPEU, S. M. A. A.; FERNANDES, F. I.; CASTRO, G. A.; LUCARELI, P. R. G.; FUKUDA, T. Y.. O efeito da eletroestimulação transcutânea na espasticidade pós acidente vascular cerebral. Neurociências, v.22, n.3, p.418424, 2014.

RODRIGUES, E. S. R.; CASTRO, K. A. B.; REZENDE, A. A. B.; HERRERA, S. D. S. C., PEREIRA, A. M.; TAKADA, J. A. P.. Fatores de risco cardiovascular em pacientes com acidente vascular cerebral. Amazônia: Science \& Health, v.1, n.2, p.21-28, 2013

SILVA, J. P.; BOUSFIELD, A. B. S.; CARDOSO, L. H.. A hipertensão arterial na mídia impressa: análise da revista Veja. Psicologia e Saber Social, v.2, n.2, p.191-203, 2014.

SIQUEIRA, A. O.; BARBOSA, R. F. M.. Terapia por Contensão Induzida e Treino Mental na Função de Membro Superior Pós-AVC. Neurociências, v.21, n.2, p.193-201, 2013.

SOUZA, A. M. L. B.; PAULA, P. A.. Acidente Vascular Isquêmico: Revisão Sistemática dos aspectos atuais do tratamento na fase aguda. Ensaios e Ciência, Ciências Biológicas, Agrárias e da Saúde, v.16, n.4, 2012.

SPENCE, J. D.. Acidente vascular cerebral: prevenção, tratamento e reabilitação. Porto Alegre: AMGH, 2013.

WISSEL, J.; MANACK, A.; BRAININ, M.. Toward an epidemiology of poststroke spasticity. Neurology, v.80, n.3, p.13-19, 2013.

A CBPC - Companhia Brasileira de Produção Científica (CNPJ: 11.221.422/0001-03) detém os direitos materiais desta publicação. Os direitos referem-se à publicação do trabalho em qualquer parte do mundo, incluindo os direitos às renovações, expansões e disseminações da contribuição, bem como outros direitos subsidiários. Todos os trabalhos publicados eletronicamente poderão posteriormente ser publicados em coletâneas impressas sob coordenação da Cognitionis Publishing, da Companhia Brasileira de Produção Científica e seus parceiros autorizados. Os (as) autores (as) preservam os direitos autorais, mas não têm permissão para a publicação da contribuição em outro meio, impresso ou digital, em português ou em tradução. 